\title{
THE DIFFERENTIABILITY OF RIEMANN'S FUNCTION
}

\section{A. SMITH}

Abstract. The function $g(x)=\sum_{p=1}^{\infty}\left(\sin \pi p^{2} x / \pi p^{2}\right)$, thought by Riemann to be nowhere differentiable, is shown to be differentiable only at rational points expressible as the ratio of odd integers. The proof depends on properties of Gaussian sums, and these properties enable us to give a complete discussion of the possible existence of left and right derivatives at any point.

Introduction. Riemann asserted that the continuous function

$$
g(x)=\sum_{p=1}^{\infty} \frac{\sin \pi p^{2} x}{\pi p^{2}}
$$

is nowhere differentiable, and Hardy [1] proved that $g$ has no finite derivative at irrational points nor at rational points of the form $2 p /(4 q+1)$ or $(2 p+1) /(4 q+2)$. Recently interest in this problem has be $n$ revived because Gerver [2] proved that $g$ has a derivative $-\frac{1}{2}$ at points of the form $(2 p+1) /(2 q+1)$. He also proved that $g$ has no finite derivative at points of the form $(2 p+1) / 2^{n}$ with $n \geqq 1$.

We now extend the results to the remaining cases, showing also the existence of finite left- and right-hand derivatives at certain rationals, and proving that these derivatives exist at all rationals if we allow the values $\pm \infty$. Since Gerver's proof is extremely long and Hardy obtains his results indirectly we give a completely elementary and fairly short proof of all the above assertions.

For convenience we work with the function

$$
f(x)=x+2 g(x)=x+2 \sum_{p=1}^{\infty} \frac{\sin \pi p^{2} x}{\pi p^{2}}
$$

so that, for example, we shall verify that $f^{\prime}(x)$ exists and is zero when $x$ is of the form $(2 p+1) /(2 q+1)$.

The following lemma is all that is required to obtain expansions of $f(x)$ about a rational point $x$, which using properties of Gaussian sums reveal the properties of the derivatives.

Received by the editors May 24, 1971.

AMS 1970 subject classifications. Primary 26A27; Secondary 10G05, 40A05, 42A20.

Key words and phrases. Riemann function, nondifferentiability, Poisson summation formula, Gaussian sums.

(c) American Mathematical Society 1972 
LEMMA 1. Let $\varphi$ be a continuous function belonging to the space $L_{1}(-\infty, \infty)$. Suppose the series for $Q(\alpha)$ defined below converges uniformly in every finite $\alpha$ interval, for each fixed $h>0$. Let

$$
\hat{\varphi}(y)=\int_{-\infty}^{+\infty} e^{-2 \pi i x y} \varphi(x) d x
$$

and suppose that $|y|^{\beta}|\hat{\varphi}(y)|$ is bounded for some fixed $\beta>1$.

Then for any real constant $\alpha$, as $h \rightarrow 0^{+}$,

$$
Q(\alpha)=\sum_{k=-\infty}^{\infty} h \varphi(h k+h \alpha)=\hat{\varphi}(0)+O\left(h^{\beta}\right) .
$$

Proof. The conditions on $\varphi$ allow us to apply the Poisson summation formula to the left-hand side of (2) to obtain

$$
\sum_{k=-\infty}^{\infty} h \varphi(h k+h \alpha)=\sum_{k=-\infty}^{\infty} e^{2 \pi i k \alpha} \hat{\varphi}\left(\frac{k}{h}\right)
$$

provided this series converges absolutely $[3, \mathrm{p} .8]$.

The condition on $\hat{\varphi}$ gives, for $k \neq 0$,

$$
\left|e^{2 \pi i k \alpha} \hat{\varphi}(k / h)\right|=O\left(h^{\beta} /|k|^{\beta}\right)
$$

which shows that the above sum, leaving out the $k=0$ term, converges absolutely and is $O\left(h^{\beta}\right)$. Thus

$$
\sum_{k=-\infty}^{\infty} e^{2 \pi i k \alpha} \hat{\varphi}(k / h)=\hat{\varphi}(0)+O\left(h^{\beta}\right) .
$$

Lemma 2. Define

$$
\begin{aligned}
\varphi_{1}(x) & =(\sin \pi x) / \pi x, & & x \neq 0, \\
& =1, & & x=0, \\
\varphi_{2}(x) & =(1-\cos \pi x) / \pi x, & & x \neq 0, \\
& =0, & & x=0 .
\end{aligned}
$$

Then Lemma 1 with $\beta=2$ applies to the functions $\psi_{i}(x)=\varphi_{i}\left(x^{2}\right), i=1,2$, and

$$
\sum_{k=-\infty}^{\infty} h \psi_{i}(h k+h \alpha)=2^{1 / 2}+O\left(h^{2}\right), \quad i=1,2
$$

Proof. The conditions on $\varphi$ follow in a straightforward manner. 
To verify that the conditions on $\hat{\varphi}$ hold, let

$$
h(y)=\hat{\psi}_{1}(y)+i \hat{\psi}_{2}(y)=\int_{-\infty}^{\infty} \frac{\exp \left(i \pi x^{2}\right)-1}{i \pi x^{2}} e^{-2 \pi i x y} d x .
$$

By a simple transformation, justified by contour integration, we obtain

$$
\begin{aligned}
h(y) & =e^{i \pi / 4} \int_{-\infty}^{+\infty} \frac{1-\exp \left(-\pi x^{2}\right)}{\pi x^{2}} \exp \left(-2^{1 / 2} \pi(i-1) x y\right) d x \\
& =e^{i \pi / 4} \int_{-\infty}^{+\infty} \int_{0}^{1} \exp \left(-\pi x^{2} t\right) d t \exp \left(-2^{1 / 2} \pi(i-1) x y\right) d x .
\end{aligned}
$$

Reversing the order of integration is easily justified and evaluating the $x$ integral gives

$$
\begin{aligned}
h(y) & =e^{i \pi / 4} \int_{0}^{1} t^{-1 / 2} \exp \left(-i \pi y^{2} / t\right) d t \\
& =e^{i \pi / 4} \int_{1}^{\infty} t^{-3 / 2} \exp \left(-i \pi y^{2} t\right) d t .
\end{aligned}
$$

Putting $y=0$ gives $h(0)=\hat{\psi}_{1}(0)+i \hat{\psi}_{2}(0)=2^{1 / 2}(1+i)$ and integrating (6) by parts gives, for $y \neq 0$,

$$
h(y)=\frac{\exp \left(i \pi / 4-i \pi y^{2}\right)}{i \pi y^{2}}+O\left(y^{-4}\right)
$$

which shows $\left|y^{2} h(y)\right|$ is bounded.

Lemma 3. Let $x=r / s,(r, s)=1$. Define

$$
G(x)=\sum_{t=0}^{s-1} \exp \left(i \pi t^{2} x\right)=C(x)+i S(x) \equiv \sum_{t=0}^{s-1} \cos \pi t^{2} x+i \sum_{t=0}^{s-1} \sin \pi t^{2} x
$$

then

(7) when $r \equiv 0(\bmod 2)$,

$$
\begin{aligned}
G(x) & =\left\{\frac{1}{2} r / s\right\} s^{1 / 2}=1, & & s \equiv 1(\bmod 4), \\
& =i\left\{\frac{1}{2} r / s\right\} s^{1 / 2}=i, & & s \equiv 3(\bmod 4) ;
\end{aligned}
$$

(8) when $s \equiv 0(\bmod 2)$,

$$
\begin{aligned}
G(x) & =\left\{\frac{1}{2} s / r\right\}(s / 2)^{1 / 2}(1+i), & & r \equiv 1(\bmod 4), \\
& =\left\{\frac{1}{2} s / r\right\}(s / 2)^{1 / 2}(1-i), & & r \equiv 3(\bmod 4),
\end{aligned}
$$

where the form $\{h / k\}$ indicates a Jacobi symbol;

(9) when $r s \equiv 0(\bmod 2)$,

$$
|G(x)|=s^{1 / 2}
$$


Proof. For (7), (8) see [3, p. 38], [4]. (9) follows immediately since the Jacobi symbol takes only the values \pm 1 .

The derivative at rationals. Let $x=r / s,(r, s)=1, r s \equiv 0(\bmod 2)$.

$$
\begin{aligned}
\frac{1}{2}\left[f\left(x+h^{2}\right)+f\left(x-h^{2}\right)\right] & =x+2 \sum_{p=1}^{\infty} \frac{\sin \pi p^{2} x}{\pi p^{2}} \cos \pi p^{2} h^{2} \\
& =f(x)-h^{2} \sum_{p=-\infty}^{\infty} \sin \pi p^{2} x \psi_{2}(p h) .
\end{aligned}
$$

Write $p=k s+t \quad(0 \leqq t \leqq s-1)$. Note $\sin \pi(k s+t)^{2} x=\sin \pi t^{2} x$, since $r s \equiv 0(\bmod 2)$. Then

$$
\begin{aligned}
\frac{1}{2}\left[f\left(x+h^{2}\right)+f\left(x-h^{2}\right)\right] & =f(x)-h^{2} \sum_{t=0}^{s-1} \sum_{k=-\infty}^{\infty} \sin \pi t^{2} x \psi_{2}(k h s+h t) \\
& =f(x)-\frac{h}{s} \sum_{t=0}^{s-1} \sin \pi t^{2} x\left[2^{1 / 2}+O\left(h^{2}\right)\right] \\
& =f(x)-2^{1 / 2} S(x) h / s+O\left(h^{3}\right)
\end{aligned}
$$

where Lemma 2 has been used in the penultimate line. Similarly

$$
\frac{1}{2}\left[f\left(x+h^{2}\right)-f\left(x-h^{2}\right)\right]=2^{1 / 2} C(x) h / s+O\left(h^{3}\right)
$$

and so we obtain

$$
f\left(x \pm h^{2}\right)=f(x)-2^{1 / 2}[S(x) \mp C(x)] h / s+O\left(h^{3}\right) .
$$

Now suppose $r s \equiv 1(\bmod 2)$. It is easy to verify the relation $f(x)=$ $1+\frac{1}{2} f(4 x)-f(x+1)$ which enables us to use (10) to deduce that

$$
\begin{aligned}
& f\left(x+h^{2}\right)=f(x) \\
& \quad-2^{1 / 2}\{S(4 x)-S(x+1) \mp[C(4 x)-C(x+1)]\} h / s+O\left(h^{3}\right) .
\end{aligned}
$$

The properties of Jacobi symbols give

$$
\left\{\frac{2 r}{s}\right\}=\left\{\frac{2 r+2 s}{s}\right\}=\left\{\frac{4((r+s) / 2)}{s}\right\}=\left\{\frac{(r+s) / 2}{s}\right\},
$$

since 4 is the square of the prime 2 and $s \equiv 1(\bmod 2)$.

This immediately simplifies (11) to

$$
f\left(x \pm h^{2}\right)=f(x)+O\left(h^{3}\right)
$$

Thus when $r \equiv s 1(\bmod 2), f^{\prime}(x)$ exists and is zero, since the right-hand derivative

$$
f_{+}^{\prime}(x)=\lim _{h^{2} \rightarrow 0} \frac{f\left(x+h^{2}\right)-f(x)}{h^{2}}
$$


and the left-hand derivative

$$
f_{-}^{\prime}(x)=\lim _{h^{2} \rightarrow 0} \frac{f(x)-f\left(x-h^{2}\right)}{h^{2}}
$$

both exist and are zero. In this case it follows that the symmetric derivative

$$
f_{0}^{\prime}(x)=\lim _{h^{2} \rightarrow 0} \frac{f\left(x+h^{2}\right)-f\left(x-h^{2}\right)}{2 h^{2}}
$$

also exists and is zero. When $r s \equiv 0(\bmod 2),(10)$ shows that $f^{\prime}(x)$ is finite if and only if $G(x)=0$. However, by Lemma $3, G(x)$ is not zero. Hence $f^{\prime}(x)$ is not finite for $r s$ even. It is easy to verify that $f_{+}^{\prime}(x)$ when $r \equiv 1$ $(\bmod 4), f_{-}^{\prime}(x)$ when $r \equiv 3(\bmod 4)$, and $f_{0}^{\prime}(x)$ when $s \equiv 3(\bmod 4)$ are all zero, but in other cases these derivatives are infinite.

Derivatives at other points. At negative rationals the results of the preceding section carry over, since $f$ is an odd function.

Suppose now $x$ is irrational, which without essential loss we take to be positive.

Let $\left\{q_{n}\right\}$ be a strictly increasing sequence of positive integers, and let $p_{n}$ be the least integer such that $x_{n}=2 p_{n} /\left(4 q_{n}+1\right)>x$. Then $x_{n}-x<$ $2 /\left(4 q_{n}+1\right)$ and $x_{n} \rightarrow x$ as $n \rightarrow \infty$. From (10) and (7),

$$
\left|\frac{f(x)-f\left(x_{n}\right)}{x-x_{n}}\right|=\left\{\frac{1}{2}\left(4 q_{n}+1\right)\left(x_{n}-x\right)\right\}^{-1 / 2}+O\left(\left(x_{n}-x\right)^{1 / 2}\right) .
$$

Thus

$$
\liminf _{n \rightarrow \infty}\left|\frac{f(x)-f\left(x_{n}\right)}{x-x_{n}}\right| \geqq 1 \text {. }
$$

Let $y_{n}=x_{n}+1 /\left(4 q_{n}+1\right)=\left(2 p_{n}+1\right) /\left(4 q_{n}+1\right)$. Then $y_{n} \rightarrow x$ as $n \rightarrow \infty$ and

$$
\lim _{n \rightarrow \infty} \frac{f(x)-f\left(y_{n}\right)}{x-y_{n}}=0 \text {. }
$$

From (13) and (14) we obtain Hardy's result that $f$ does not have a finite or infinite derivative at the irrational point $x$.

Acknowledgements. I am indebted to two of my colleagues, P. R. Beesack for bringing this problem to my notice and for his criticisms of an earlier draft, and K. S. Williams for assistance in the number theory results. 


\section{REFERENCES}

1. G. H. Hardy, Weierstrass's non-differentiable function, Trans. Amer. Math. Soc. 17 (1916), 301-325.

2. J. Gerver, The differentiability of the Riemann function at certain rational multiples of $\pi$, Amer. J. Math. 92 (1970), 33-55. MR 42 \#434.

3. R. E. Bellman, A brief introduction to theta functions, Athena Series: Selected Topics in Math., Holt, Rinehart and Winston, New York, 1961. MR 23 \#A2556.

4. E. Landau, Elementary number theory, Chelsea, New York, 1958. MR 19, 1159.

Department of Mathematics, Carleton University, Ottawa, Ontario, Canada 